\title{
Behavior of Fish Retinal Growth Cones Encountering Chick Caudal Tectal Membranes: A Time-Lapse Study on Growth Cone Collapse
}

\author{
Martin Bastmeyer * and Claudia A. O. Stuermer \\ Faculty of Biology, University of Konstanz, P.O. Box 7750, Konstanz, Germany
}

\section{SUMMARY}

In a cross species in vitro assay, growth cones from fish temporal retina elongating on laminin lanes were observed with time-lapse videomicroscopy as they encountered lanes and territories that carried membrane fragments from the chick caudal tectum. Caudal tectal membranes of adult fish and embryonic chick are known to possess a repellent guiding component for temporal retinal axons. The caudal membranes of chick exert a particularly strong influence on fish temporal axons. Contacts with chick caudal membranes by just a few filopodia and parts of the lamellipodia evoked a turning response away from the membrane lane of the entire growth cone. Contacts by filo- and lamellipodia over the entire circumference of the growth cone, however, caused invariably growth cone collapse and retraction. During growth cone turning and collapse and retraction, filopodia remained in contact with the tectal membrane fragments, suggesting strong membrane-filopodia adhesion simultaneous to growth cone repulsion by the repellent guiding component. (C) 1993 John Wiley \& Sons, Inc.

Keywords: goldfish, regenerating axons, axonal guidance, in vitro assay, videomicroscopy.

\section{INTRODUCTION}

The establishment of orderly nerve cell connections depends on the ability of the growth cone to read and respond to cues in its local environment. A large number of molecules has been defined that exert growth-promoting influences on the growth cones such as extracellular matrix (ECM) molecules, laminin being one of them, and certain cell adhesion molecules such as neural cell adhesion molecule (N-CAM), members of the L1 family and several others (for review: Jessell, 1988). More recently, however, molecular cues with growth-inhibiting or repellent influences have been identified (for review: Davies and Cook, 1991; Patterson, 1988).

One of these molecules with axon repellent influ- ence is likely to be involved in guiding retinal axons in vivo to their retinotopic target sites in the tectum. It is graded in its distribution with its maximum in concentration over the most posterior (caudal ) part of the tectum and declining towards the anterior (rostral) tectal pole (Walter et al., 1987a,b; Baier and Bonhoeffer, 1992). The axonguiding function of this molecule in the chick retinotectal system has been revealed by an in vitro stripe assay (Walter et al., 1987a). In this assay, membranes of embryonic chick rostral and caudal tectal membranes are applied side by side in narrow alternating stripes and this striped carpet is offered as a substrate to chick temporal and nasal retinal axons. In this assay temporal axons accumulate on rostral membrane stripes and avoid the caudal membranes (Walter et al., 1987b).

Applying this assay to other species, a similar segregation of axons in response to the caudal tectal membranes was discovered in adult fish (Vielmetter and Stuermer, 1989) and embryonic mouse (Godement and Bonhoeffer, 1989). The molecule of caudal tectal membranes with axon-repellent in- 
fluence is probably evolutionary conserved. This conclusion derived from cross-species in vitro stripe assays, in which fish temporal axons were exposed to alternating stripes of rostral and caudal membranes of embryonic chick tecta (Vielmetter, Walter, and Stuermer, 1991a). Fish temporal axons accumulated on the stripes of chick rostral membranes. Embryonic mouse axons on chick tectal membranes exhibited a similar response (Godement and Bonhoeffer, 1989).

In the cross-species assay with chick membranes and fish axons, we noted that the fish temporal axons responded to the chick caudal membranes much more strongly than to their own, the fish caudal membranes (Vielmetter et al., 1991a). On a striped carpet of fish rostral and caudal membranes, fish temporal axons occasionally crossed the caudal membrane stripes, but they were unable to pass chick caudal membranes. Moreover, the temporal fish axons were clustered in the very center of the chick rostral membrane stripes keeping at distance to the chick caudal membrane territories.

In the present study we were interested in analyzing the response of individual growth cones when they meet the boundaries of the caudal membrane stripes with time-lapse videomicroscopy. We analyzed, in particular, the encounter of fish temporal axons with chick caudal membranes, because in this situation, the fish growth cones exhibited a dramatic avoidance response and even collapsed.

To be able to monitor the growth cone behavior at membrane borders with time-lapse videomicroscopy we modified the stripe assay. This modification allowed us to observe the growth cones with phase contrast at a high magnification for extended periods of time. It is also possible to observe growing axons on striped membrane carpets with two types of membranes in adjacent stripes when axons are stained with fluorescent dyes (for instance with carbocyanine dyes or rhodamine isothiocyanate) (Müller, Stahl, and Bonhoeffer, 1990), however, long-lasting illuminations of the dye-labeled axons often causes axon degeneration and collapse as photodamage artifacts (Kaethner and Stuermer, 1992). In the modified stripe assay used here only one type of membrane, either from rostral or caudal tectum was used and applied in stripes to a laminin-coated coverslip with the aid of a special silicon matrix (Vielmetter, Stolze, Bonhoeffer, and Stuermer, 1990). Axons elongating on the laminin lane were observed as they made contacts with the membrane particles in adjacent lanes and at the end of the lanes where a continuous membrane boundary is present. Growth cones extending on the laminin lane were seen to touch the membrane particles with their filopodia and with the tips of narrow lamellipodial extensions. With caudal chick membranes these contacts were sufficient to cause a turn of the growth cone away from the membrane boundary. At the end of the lane where growth cones are surrounded by membranes on three sides, they failed to advance, collapsed, and retracted, but left behind strands of filopodia adhering to the membrane particles. Preliminary accounts of this work were included in conference reports (Stuermer, 1989; Stuermer, Bastmeyer, and Vielmetter, 1990) and television programs (ZDF, WDR, BBC) and in Spektrum Videothek (1990).

\section{METHODS}

A fraction enriched in cell surface membranes of either the rostral or caudal tectal third from embryonic chick [embryonic day (E) 13] and adult goldfish were prepared as previously described (Walter et al., 1987a,b; Vielmetter and Stuermer, 1989). The membranes were applied onto a poly-lysine/laminin-coated coverslip in stripes with a special silicon matrix. Coating of the coverslip and a detailed description of the matrix were presented earlier (Vielmetter et al., 1990). In brief, the matrix consists of a parallel array of open channels and bars. At the ends the channels merge into an inlet and outlet channel which are perpendicular to the parallel channels. The bars are in contact with the coverslip and protect the coverslip from the membranes which are applied [5-10 $\mu$ l of membranes in phosphate-buffered saline (PBS), $500 \mu \mathrm{g} / \mathrm{ml}$, determined by optical density] through a syringe into the inlet channel and spread through the entire channel system. Through centrifugation at $3000 \mathrm{~g}$ for $15 \mathrm{~min}$ the membranes are made to adhere to the coverslip. Thereafter, the silicon matrix is removed and a patterned substrate of laminin lanes alternating with membrane lanes is created. The laminin lanes are bounded at both ends by nonstriped membrane-covered territories (Fig. 1). An explant from either the temporal or nasal retina of adult goldfish, 14-16 days after optic nerve section, are prepared as reported (Vielmetter and Stuermer, 1989) and placed perpendicular to the parallel lanes onto the coverslip. Thus, axons growing out from the explant meet repeatedly the borders between the laminin and membrane lanes and are at the end of the lanes confronted with a homogeneous membrane-covered territory (Fig. 1). The preparations were kept in L-15 [Leibowitz medium (Gibco) supplemented with $10 \%$ fetal calf serum (FCS), $0.4 \%$ meth- 
ylcellulose and $50 \mu \mathrm{g} / \mathrm{ml}$ gentamycin ] at $22^{\circ} \mathrm{C}$ in petriperm dishes (Heraeus).

The growth of axons was viewed in daily intervals up to 5 days after retina explantation. Selected preparations were used for time-lapse recordings of growing axons. Living axons were monitored in phase contrast with a $40 \times$ objective using an inverted microscope (Zeiss IM$35)$ to which a camera was attached. The camera was connected to an image processor (Hamamatsu) and a U-matic recorder (Sony) coupled to a time-lapse system (EOS). To avoid continuous illumination, we inserted a shutter into the light path which opened every $15 \mathrm{~s}$ for $200 \mathrm{~ms}$. Four images were taken at once and averaged and recorded.

For immunofluorescence, the preparations were fixed first in methanol $\left(-20^{\circ} \mathrm{C}\right)$ and then in $2 \%$ paraformaldehyde in PBS for 5 min each. Antibodies and their concentrations were as follows: Primary antibodies were monoclonal antibody (MAB) L2/HNK-1 (kindly provided by $M$. Schachner) diluted 1:100 in PBS, and MAB E 587 (Vielmetter et al., 1991b) at a concentration of $100 \mu \mathrm{g} / \mathrm{ml}$. After $1 \mathrm{~h}$ incubation at room temperature and three washes in PBS, the preparations were exposed to secondary antibodies [fluorescein isothiocyanate (FITC) or rhodamine-coupled goat anti-mouse or antirat ] for $1 \mathrm{~h}$, washed in PBS, and coverslipped under Moviol. Antibody-labeled preparations were viewed and photographed with $100 \times$ lenses at a Zeiss Axiophot equipped with the appropriate fluorescence filter sets.

\section{RESULTS}

\section{Avoidance of Chick Caudal Membranes}

Axons from goldfish temporal retina react to the repellent-guiding component of embryonic chick caudal tectal membranes more strongly than to the component on the fish caudal tectal membranes. This result obtained in an earlier study (Vielmetter et al., 1991a) in which axons were exposed to alternating stripes of rostral and caudal tectal membranes was confirmed in the present modified in vitro stripe assay. As illustrated in Figure 1, temporal axons were restricted to the uncovered laminin lanes and did not cross the lanes carrying chick caudal membranes, nor did they penetrate into the membrane-covered territory at the end of the lanes. The photomicrograph in Figure 1 was taken from a preparation 5 days after retinal explantation.

The accumulation of the axons on the laminin lanes was not the result of a physical restriction by the membrane particles since fish nasal axons crossed frequently over the chick caudal mem- brane lanes and also penetrated into the membrane territory at the ends (Fig. 2). When membranes were derived from chick rostal tectum, both temporal (Fig. 3) and nasal axons (not shown) grew freely over the membrane territories.

Figure 1(B) shows a higher magnification of fish temporal axons at the ends of the laminin lanes where they are surrounded by chick caudal membranes from three sides. Growth cones were no longer discernible. The axons were heavily fasciculated and exhibited a beaded appearance. Numerous filopodial strands extended between the axons and the membrane fragments. The filopodial strands and the abnormal axon morphology were specific for the situation in which fish temporal axons encountered chick caudal membranes.

\section{Dynamics of Growth Cone Repulsion}

Time-lapse video observations on individual growth cones revealed the dynamics of growth cone membrane interactions. As axons emerging from the retinal explant extended on the laminin lane they had large growth cones with ruffling veils and numerous filopodia as common for goldfish growth cones on a substrate of laminin (Vielmetter et al., 1990) and progressed with an average velocity of about $100 \mu \mathrm{m} / \mathrm{h}$ (Table 1). When axons either from nasal or temporal (Fig. 3) goldfish retinal explants met membranes from the rostral chick tectum most growth cones grew onto the membranes and continued their growth without changing their velocity significantly (Table 1). Nasal goldfish axons ( six out of eight, Table 1) were able to elongate on chick caudal membranes, but their growth velocity was reduced to about $30 \mu \mathrm{m} / \mathrm{h}$ ( Table 1).

The situation changed dramatically when temporal axons interacted with caudal membranes. While elongating on the laminin lanes, most growth cones made repeated contacts with the membranes to the left and right of their laminin path (Fig. 4). These contacts were typically made either with what appeared to be filopodia only or lamellipodial veils that spread between the filopodia. Such membrane encounters with a fraction of the growth cones circumference was sufficient to evoke a turning response of the growth cone away from the sites of membrane contact or to elicit branching of the growth cone and retraction of the lamellipodia that had touched the membranes [Fig. 4(A-D)]. After turning most growth cones interacted with the membrane particles on the 

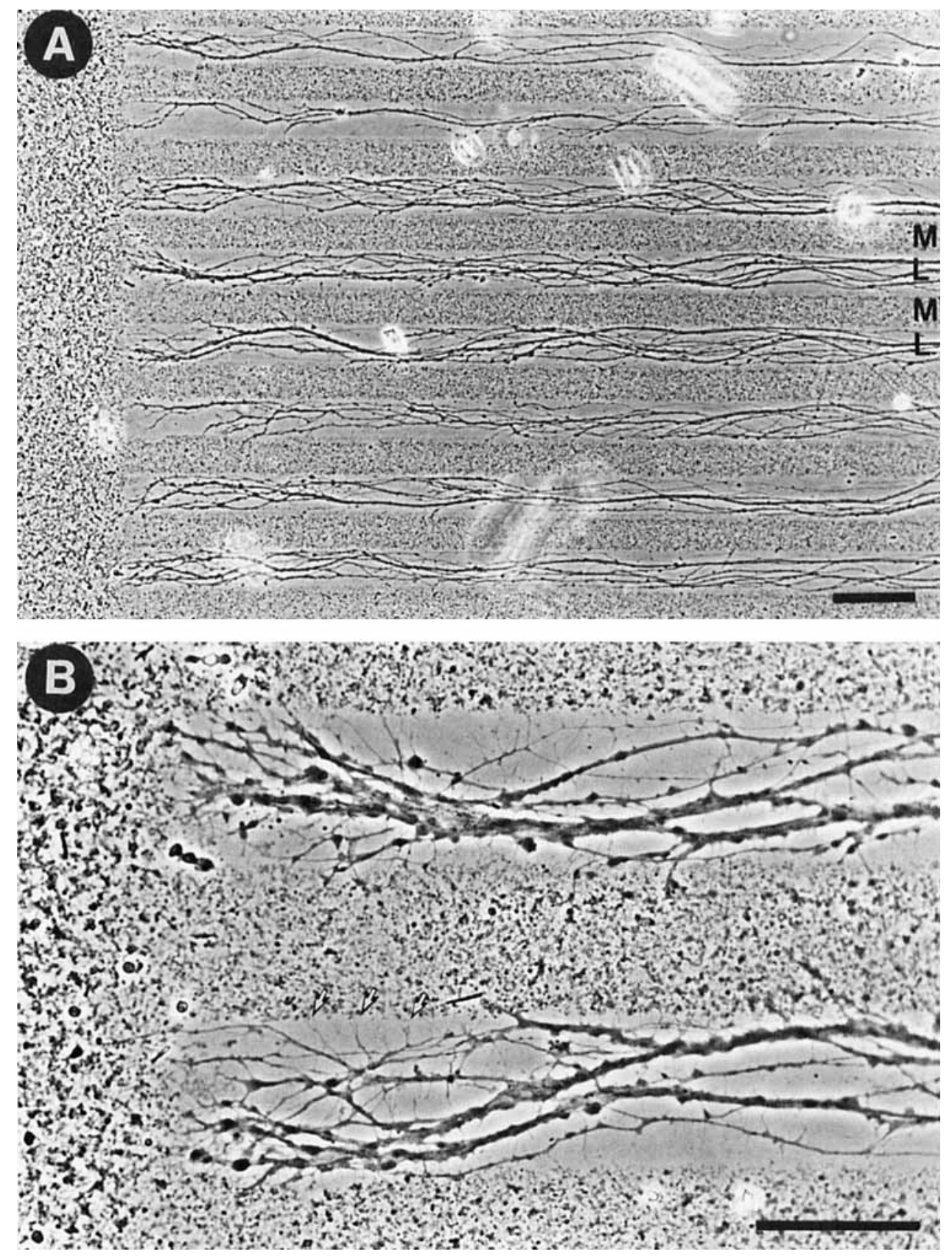

Figure 1 Temporal axons and caudal membranes. Phase-contrast micrograph of a living preparation of goldfish temporal retinal axons growing on a patterned substrate of alternating lanes of polylysine/laminin ( $\mathrm{L}$ ) and chick caudal tectal membranes ( $\mathrm{M}$ ) after 5 days in vitro. The retinal explant is to the right. (A) The axons grow almost exclusively on the laminin lanes. Note the sinosoidal axonal growth pattern. (B) Higher magnification showing numerous fasciculated axons at the ends of the laminin lanes. The fascicles have a beaded appearance and growth cones are not discernable. Scale bars: A, $100 \mu \mathrm{m} ; \mathrm{B}, 50 \mu \mathrm{m}$.

other side of the lane before they were again repelled [Fig. 4(D-I)]. This behavior is reflected in the sinosoidal growth pattern seen in low power micrographs (Fig. 1).
Surprisingly, however, many filopodia remained attached to the membrane particles even after the growth cone had turned away. Simultaneously, a remarkable backward movement of 


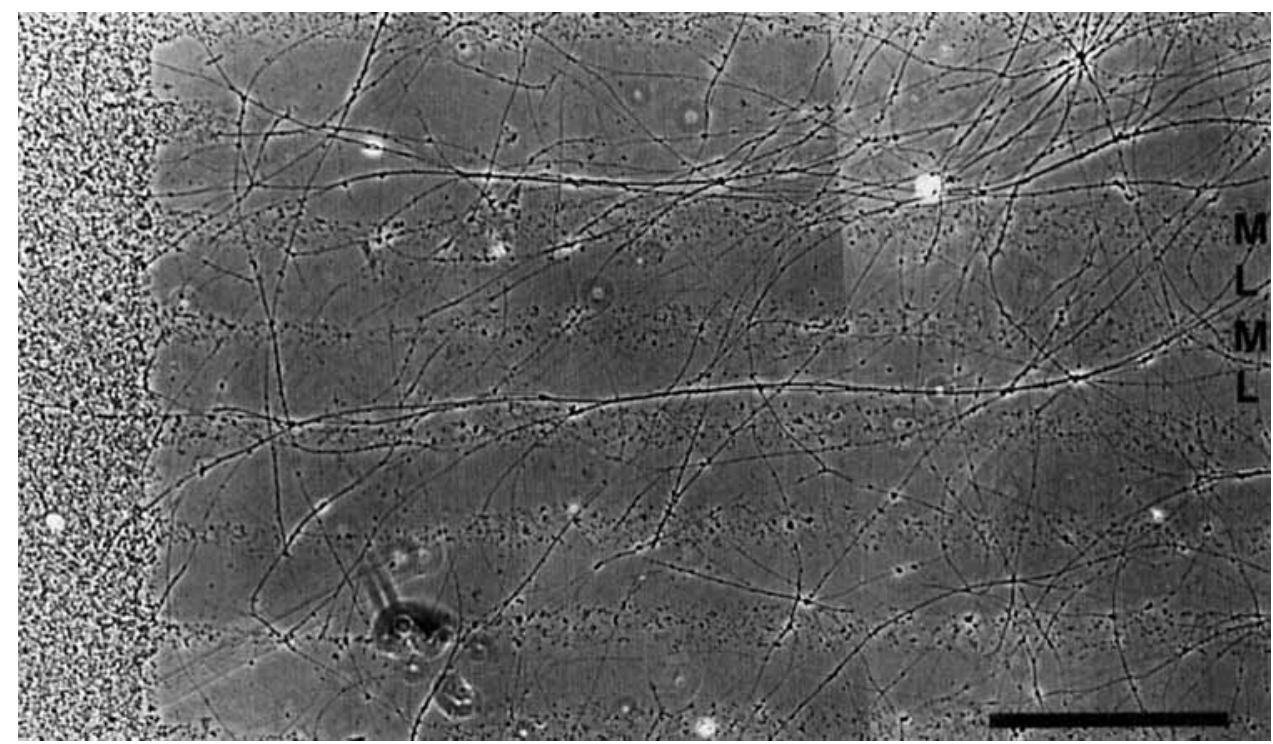

Figure 2 Nasal axons and caudal membranes. Phase-contrast micrograph of a living preparation of goldfish nasal retinal axons growing on a patterned substrate of alternating lanes of polylysine/laminin ( $L$ ) and chick caudal tectal membranes $(M)$ after 5 days in vitro. There is a slight preference of the axons for the laminin lanes, but many axons have crossed the membrane lanes and penetrated into the membrane covered territory at the end of the laminin lanes. Scale bar $=200 \mu \mathrm{m}$.

small particles commenced over each filopodium that persisted while the growth cone turned. Such backward movement was also observed over filopodia further distal from the growth cone. These filopodia had remained in contact with the membranes during earlier growth cone / membrane contacts. While backward particle movement over filopodia on or close to the growth cone began when
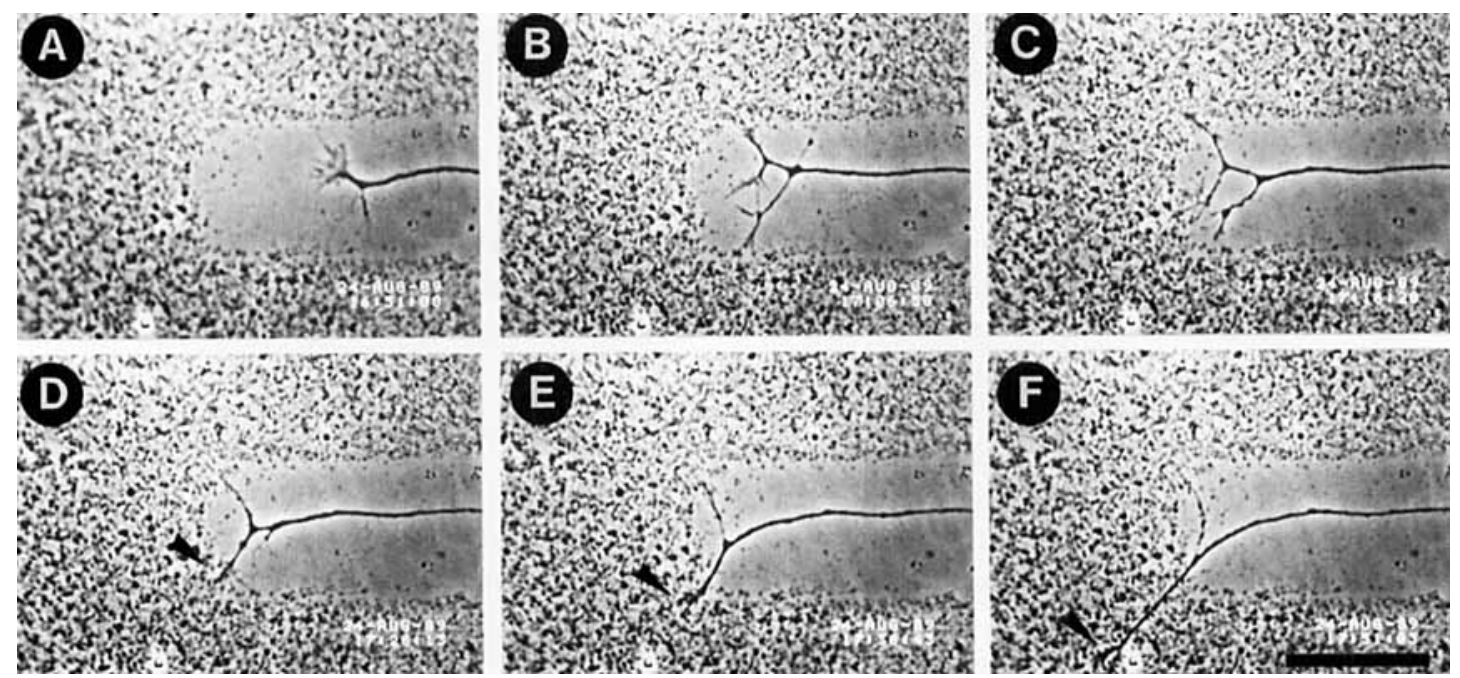

Figure 3 Temporal axons and rostral membranes. Sequence of videomicrographs of goldfish temporal retinal axons interacting with chick rostral tectal membranes. As the growth cone approaches the end of the laminin lane it branches out. It progresses with one of its branches into the membrane-covered territory (arrowheads in D-F) and retracts the other branches. Time in hours, minutes, and seconds is shown in the lower right-hand corner. Scale bar $=50$ $\mu \mathrm{m}$. 
Table 1 Response of Individual Goldfish Retinal Axons upon Contact with Chick Tectal Membranes Monitored with Time-Lapse Videomicroscopy

\begin{tabular}{lccccc}
\hline $\begin{array}{c}\text { Retinal } \\
\text { Explant/Tectal } \\
\text { Membranes }\end{array}$ & Naxons & Collapse & Advance & & \multicolumn{2}{c}{ Laminin } & Membranes \\
\cline { 3 - 6 } Temporal/rostral & 12 & 2 & 10 & $99 \pm 10$ & $98 \pm 12$ \\
Nasal/rostral & 8 & 0 & 8 & $95 \pm 17$ & $84 \pm 12$ \\
Temporal/caudal & 27 & 25 & 2 & $96 \pm 8$ & $--^{1}$ \\
Nasal/caudal & 8 & 0 & $6^{2}$ & $97 \pm 7$ & $32 \pm 7$ \\
\hline
\end{tabular}

\footnotetext{
${ }^{1}$ Two temporal axons grew onto the caudal membranes whereupon one axon stalled. The other axon continued to grow on the membranes without even changing its growth velocity.

${ }^{2}$ Two nasal axons performed a $\mathbf{U}$-turn upon contact with caudal membranes and grew on the laminin stripe back into the direction of the retinal explant.
}

the growth cone turned, particle movement events on "older" filopodia up to $200 \mu \mathrm{m}$ distal from the growth cone (within the field of view) were unpredictable, but did occur consistently and in elevated frequency during growth cone collapse. The translocation of one of such particles is illustrated in the sequence of pictures in Figure 5. This particle is clearly visible even in still images since it is relatively large. The movement of smaller particles seen in the time--lapse movies is difficult to demonstrate in still photographs, but occurred with the same velocity as that of the larger particles. Backward movement of particles was in the same time range, whether the growth cone turned away from the membrane particles and continued to grow on laminin or whether it contacted membranes with its entire circumference and collapsed (see below). The overall velocity of particle backward movement was $10.1 \pm 2.2 \mu \mathrm{m} / \mathrm{min}(n=15$, range: $7-15$ $\mu \mathrm{m} / \mathrm{min}$ ). We compared the particle movement of persisting filopodia to backward particle movement in lamellipodia and filopodia of advancing growth cones and found that the velocity of the latter two was similar to the former. In lamellipodia of advancing growth cones, particles moved with an average velocity of $9.4 \pm 1.4 \mu \mathrm{m} / \mathrm{min}(n$ $=10$, range: $8-12 \mu \mathrm{m} / \mathrm{min}$ ) in the backward direction and in their filopodia with $10.4 \pm 1.4 \mu \mathrm{m} / \mathrm{min}$ ( $n=10$, range: $9-13 \mu \mathrm{m} / \mathrm{min}$ ). Thus, backward particle movement appears to be a normal phenomenon during growth cone advance. It continues to occur in the situation in which filopodia persist upon contact with a strong repellent component.

\section{Persistant Filopodia}

During this backward movement of particles, the filopodia became for unknown reasons phase dark.
Because of the retention of the filopodia, growth cones making contacts with membrane fragments appeared to possess a larger number of filopodia than growth cones advancing on the laminin substrate without membrane contacts.

We have analyzed this filopodial behavior in more detail. Filopodia of growth cones of goldfish retinal axons emerged with a velocity of $10.6 \pm 4.3$ $\mu \mathrm{m} / \min (n=15$, range: $5-20 \mu \mathrm{m} / \mathrm{min})$ and retracted with $8.25 \pm 3.3 \mu \mathrm{m} / \mathrm{min}(n=15$, range: $3-11 \mu \mathrm{m} / \mathrm{min}$ ). Growth cones that advanced on laminin without making contacts to membrane fragments had filopodia that persisted for $4.5 \pm 3.8$ $\min (n=10$, range: $2-15 \mathrm{~min}$ ) before they retracted [arrowheads in Fig. $4(F, G)$ ]. When filopodia of temporal growth cones contacted chick rostral membranes they remained for $10 \pm 3.2 \mathrm{~min}$ ( $n=15$, range: $5-15 \mathrm{~min}$ ), however, when they contacted chick caudal membranes, filopodia of temporal axons persisted much longer, on average $70 \pm 31 \mathrm{~min}(n=15$, range: $30-120 \mathrm{~min})$, although the lamellipodia withdrew rapidly [arrows in Fig. 4(A-H)]. This suggests a strong filopodiamembrane adhesion despite of and simultaneous to growth cone repulsion.

\section{Dynamics of Growth Cone Collapse}

For some temporal growth cones, brief encounters with the caudal membrane particles on one side were sufficient to induce growth cone collapse. Most growth cones, however, collapsed in the end of the lanes. Here, the growth cone met membranes on three sides, such that the growth cone touched membranes with filopodia and lamellipodia over most of its circumference (Fig. 6). Growth cone collapse in these cases was induced within 10 $\pm 6.4 \mathrm{~min}(n=15$, range: $5-25 \mathrm{~min})$ after initial contact with the caudal membranes. The behavior 

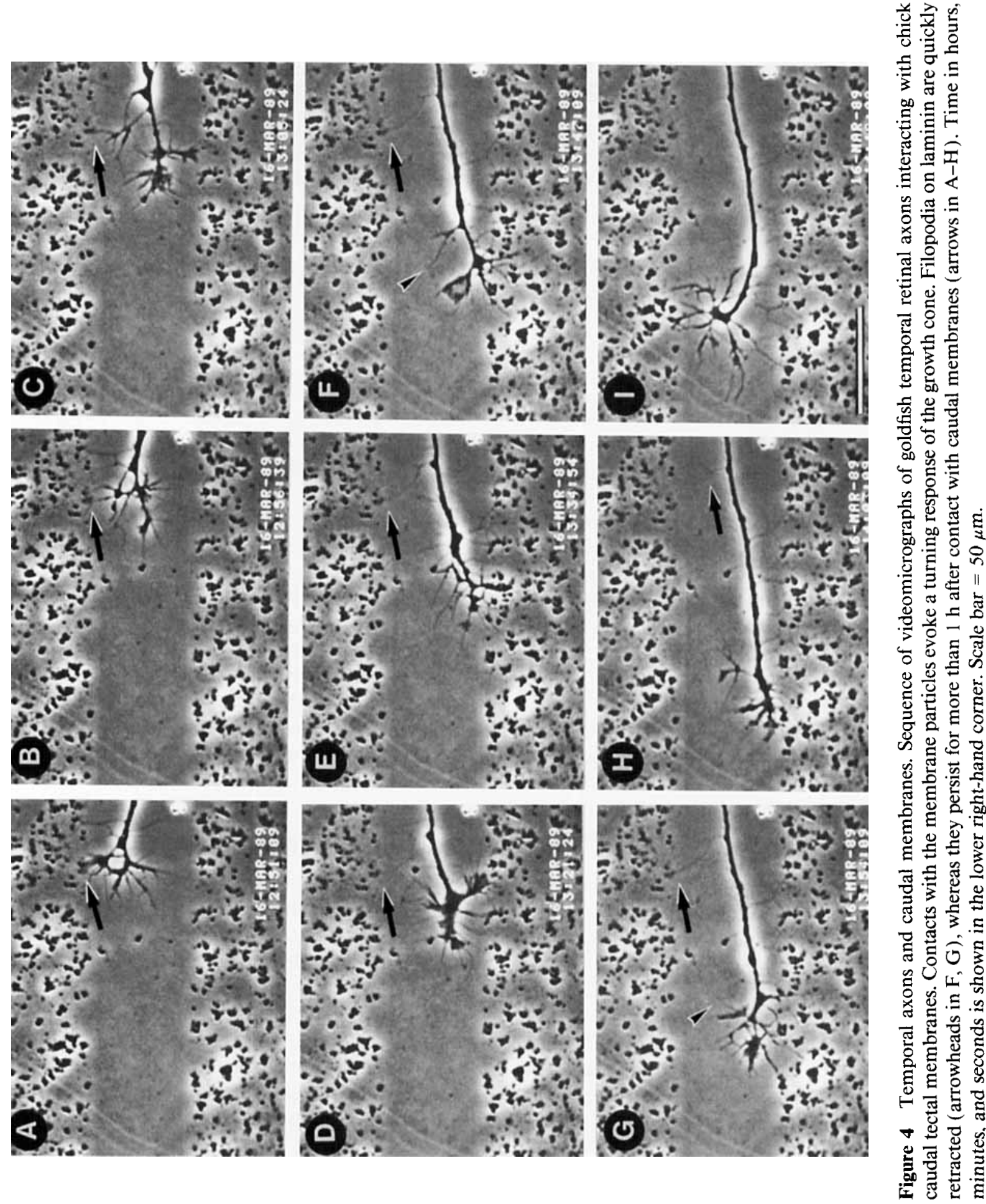

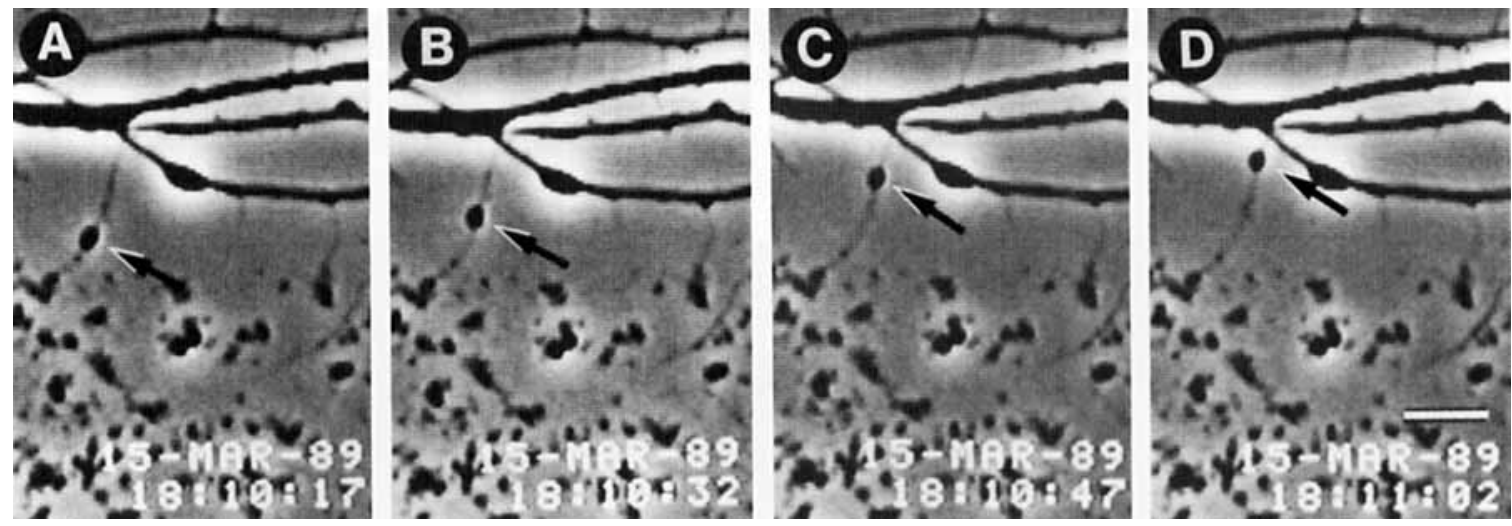

Figure 5 Backward movement of particles. Sequence of videomicrographs exemplifying the translocation of a particle (arrows in A-D) in a persisting filopodium from the filopodial tip to its base. Time in hours, minutes, and seconds is shown in the lower right-hand corner, Scale bar $=10 \mu \mathrm{m}$.

of the growth cone shown in Figure 6 was typical in these "dead end"-like situations. The growth cone touched membranes first with one side, again only with some filopodia and lamellipodia and while lamellipodia withdrew it contacted membranes in front with its forwardly extended filopodia and tips of lamellipodia. Here upon the growth cone bent only to meet membranes again on the other side. During this turning response the lamellipodia were quickly withdrawn but the filopodia at the previous contact sites remained. As filo- and lamellipodia around the growth cones circumference had touched membrane particles, the growth cone's core condensed, that is, it acquired under phasecontrast optics a darker appearance. Backward movement of growth cone material down to the axon shaft commenced and proceeded rapidly. For four growth cones that were monitored as individuals during their collapse the retraction of axoplasma was $4,6,7$, and $8 \mu \mathrm{m} / \mathrm{min}$, respectively. Particle movement into the backward direction was also seen over all filopodia including those that had been formed minutes ago and that had stayed connected to the axon shaft [Fig. $6(\mathrm{~K}-\mathrm{L})]$. Accompanied by what appeared to be ruffling of its membranes the axon retracted rapidly out of the field of view. A large number of the filopodial strands were still connected to the membrane fragments and strands of material were also left behind where the growth cone core had been on the laminin lane before it collapsed (Fig. 6).

Growth cone collapse was only seen when fish temporal axons met chick caudal membranes. When the membranes derived from fish caudal tectum the temporal axons showed a slight ten- dency to remain on the laminin lane but they also crossed the lanes and invaded into the membrane territory at the end of the lanes.

These observations show that chick caudal tectal membranes are not only nonpermissive substrates for goldfish temporal axons but that their repellent influence leads almost always to growth cone collapse (Table 1). Even after 5 days, in the oldest cultures that were examined, we hardly found growth cones that would have grown into the membrane territories. Thus, most, if not all of the axons with abnormal appearance that were clustered in the dead end of the lanes in Figure 1 are probably the remnants of axons whose growth cones had collapsed.

\section{Cell Adhesion Molecules on Persisting Filopodia}

To test whether filopodial strands would possess and retain cell surface membranes even after growth cone collapse, we used two antibodies against epitopes on cell surfaces, that is, cell adhesion molecules. This also could give a first hint whether filopodial strands and their attachment sites on chick caudal membranes may carry cell adhesion molecules. Thus, we exposed fixed preparations to the antibodies $\mathrm{L} 2 / \mathrm{HNK}-1$ and $\mathrm{E} 587$. MAB L2/HNK-1 is directed against a carbohydrate epitope associated with several cell adhesion molecules of the immunoglobulin- (Ig) superfamily and this epitope itself is involved in adhesion (Künemund et al., 1988). MAB E 587 is directed against a candidate cell adhesion molecule in the fish central nervous system (CNS) belonging to the 

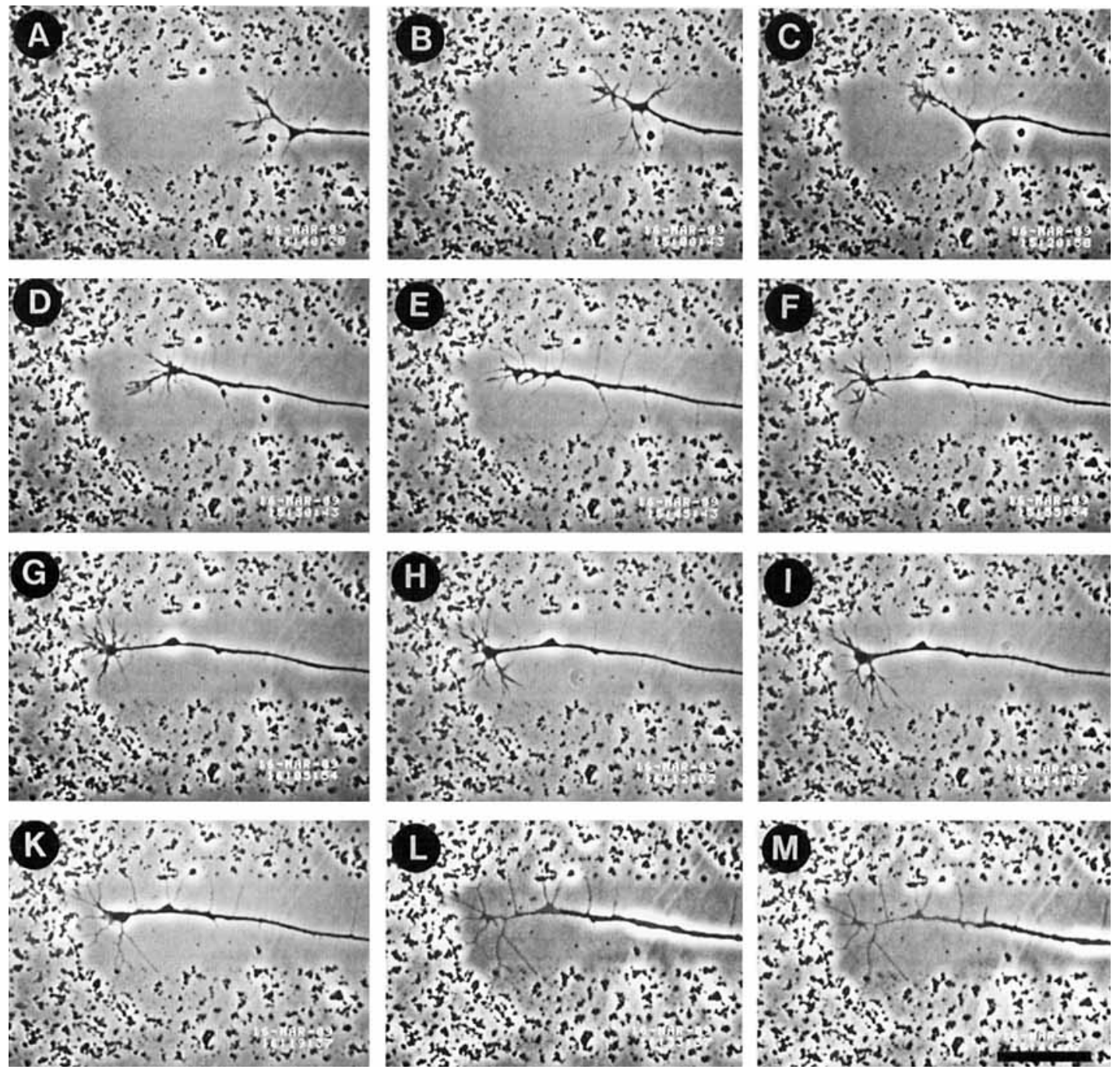

Figure 6 Temporal axons and caudal membranes. Sequence of videomicrographs of goldfish temporal retinal axons interacting with chick caudal tectal membranes. Growth cone collapse commences after extensive contacts with membrane particles in the end of the laminin lane $(\mathrm{H}, \mathrm{I})$. Note the axonal remnants after growth cone collapse ( $\mathrm{L}, \mathrm{M})$. Time in hours, minutes, and seconds is shown in the lower right-hand corner. Scale bar $=50 \mu \mathrm{m}$.

L1 family (Vielmetter, Lottspeich, and Stuermer, $1991 \mathrm{~b}$ ) and does not cross-react with other species. HNK1 stained the filopodial strands throughout their length and also stained the chick membrane particles [Fig. 7(A,B)], indicating that filopodialmembrane contacts could potentially be mediated by the HNK-1 epitope or by other epitopes on cell adhesion molecules that carry the HNK-1 epitope. E 587 antibody staining was seen on the axons and their remnants as well as on some of the filopodial strands but it did not label the chick membranes [Fig. 7(C,D)]. These observations suggest, first, that filopodial strands retain cell surface membranes and cell adhesion molecules, and second, open the possibility that filopodia-membrane contacts are mediated by cell adhesion molecules.

\section{DISCUSSION}

This time-lapse videomicroscopy study has demonstrated the dynamics of growth cone retraction and collapse as a result of the interaction of growth cones from goldfish temporal retina with caudal tectal membranes of the chick. While elongating on laminin lanes the growth cone made contacts with membrane fragments in the neighboring lanes. Local membrane contacts with just a few 

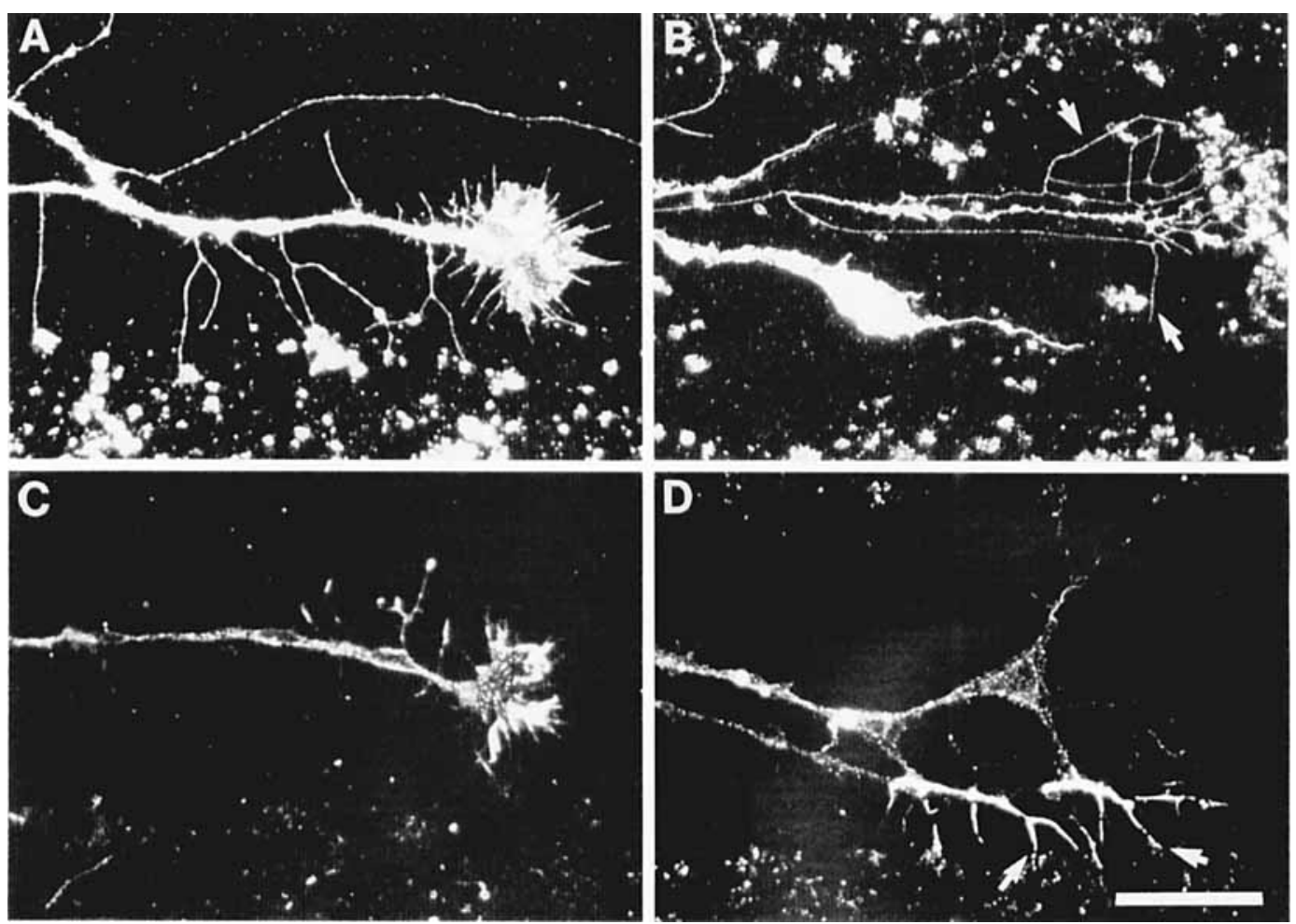

Figure 7 Temporal axons and caudal membranes. Immunofluorescence micrographs of goldfish temporal retinal axons on a patterned substrate of laminin and chick caudal membranes. Both, axons and actively elongating growth cones ( $A, C$ ) but also persisting filopodia after growth cone collapse (arrows in B, D) are positive for MAB L2/HNK-1 (A, B) and MAB E $587(\mathrm{C}, \mathrm{D})$. Chick membrane particles are only stained with Mab L2/HNK-1. Scale bar $=20 \mu \mathrm{m}$.

filopodia and narrow lamellipodial extensions on one side of the growth cone's circumference were sufficient to induce the growth cones to turn away from the membrane boundary but contacts with filopodia and tips of lamellipodial extensions over a larger extent of the growth cone's circumference elicited growth cone collapse and retraction. During growth cone collapse and retraction or turning, the filopodial tips remain attached to the membrane fragments, suggesting that strong adhesive interactions may foster these contacts.

The caudal membranes of fish and chick have been shown to contain a repellent guiding component that on a carpet of alternating stripes of rostral and caudal membranes leads to the accumulation of temporal axons on the rostral membranes and to the avoidance of caudal membranes (Walter et al., 1987a,b; Vielmetter and Stuermer, 1989). Earlier cross-species studies using the in vitro assay with striped membrane carpets have indicated that the repellent guiding component on fish and chick tecta are closely related molecules (Vielmetter et al., 1991a). Arguments for their relatedness were, first, that the repellent guiding component of both species predominantly affects one group of retinal axons, the temporal ones, and second, that influence on temporal axons was lost after treatment of the membranes with the enzyme Pi-PLC. This was, in fact, also confirmed here in the modified stripe assay, in that fish temporal axons as well as nasal axons crossed the caudal membrane stripes freely when these membranes had been subjected to PiPLC treatment prior to use in the assay (data not shown). However, the concentration of this molecule appears to be higher in embryonic chick as opposed to adult fish tecta, or else fish axons have a higher sensitivity to the chick component than to their own (Vielmetter et al., 1991a).

The same assumption must be made here to account for the reaction of the fish temporal axons in the present modified stripe assay. Growth cone collapse was the rule when fish temporal axons extending on a laminin lane met chick caudal membranes. That the collapse response is in the situation of the modified stripe assay specifically induced by axon contacts with chick caudal mem- 
branes is shown by the fact that collapse was rare when temporal axons touched caudal membranes of the fish tectum, and that neither chick or fish rostral membranes elicited growth cone retraction. Instead axons grew freely over these membrane types.

Chick caudal membranes evoked collapse also of chick temporal axons in the so-called collapse assay (Cox, Müller, and Bonhoeffer, 1990), in which liposomes loaded with the repellent component are added to the culture medium and thereby are seeded on the growth cone while it elongates on a laminin-coated surface. It was suggested that this response is the result of a sudden exposure of the "naive" growth cone, not having experienced the repellent component before, to high concentrations of this repellent component (Walter et al., 1990). Growth cones in vivo, that is, in the tectum, probably deal with subtle concentration differences which the growth cone may, in its interior, amplify to a steep gradient (Gierer, 1987). Cessation of growth and induction of collapse upon exposure to the concentrated repellent component may be due to an overreaction of the amplification, as was suggested by Walter, Allsopp, and Bonhoeffer, (1990). The modified in vitro stripe assay may represent a similar situation insofar as (1) the membranes form a sharp boundary to the laminin lanes and (2) the chick repellent component exerts a strong influence on fish axons. However, the present situation differs from the previously described collapse assay (Cox et al., 1990) in that it allows to observe the behavior of the growth cone and the dynamics of its lamelli- and filopodia interactions with the membrane fragments in its surrounding.

Chick axons were reported to be able to adapt to the repellent component, because when the caudal membranes were offered as the sole substrate and no choice was offered, chick temporal axons did grow and elongated with a velocity similar to the one found on rostral membranes (Walter et al., 1990). Fish temporal axons, however, may not be able to ever adapt or overcome the influence of the chick caudal membranes. In an outgrowth assay described earlier, axonal outgrowth failed entirely on a substrate of chick caudal membranes (Vielmetter et al., 1991a). In the dead end situation of the present study, axons would never cross or progress into the membrane territory at least not over the 5 days over which we have observed them.

Fish nasal axons are also sensitive to the repellent component of the chick caudal tectum, although to a lesser degree than fish temporal axons (Vielmetter et al., 1991a). Consistent with these findings, fish nasal axons grew onto the chick caudal membranes, but reduced their growth velocity (Table 1).

Thus, the repellent component appears to irreversibly shut down the temporal growth cone's growth program (Cox et al., 1990). The repellent component received over the tips of the filo- and lamellipodia is, therefore, dominant over the growth-stimulating effects that the ECM molecule laminin exerts on the growth cone and is dominant over the growth-promoting properties that the tectal membranes should possess. Indeed the tectal membranes whether from rostral or from caudal tectum must carry growth-supporting molecules. This is inferred from finding axon growth on rostral membrane carpets and on caudal membranes after removal of the repellent component by PiPLC (Walter et al., 1987b; Vielmetter et al., 1991a). Chick tectal cells express N-CAM on their surface (Schlosshauer, Schwartz, and Rutishauser, 1984) and are likely to carry further cell adhesion molecules, which are known to support axonal growth, the presence of which is inferred from the L2/HNK-1 positivity of the membranes. Furthermore, as shown here the membranes are L2/ HNK-1 immunoreactive, and this antibody defines a carbohydrate epitope that is found on several cell adhesion molecules of the IG superfamily (Norhona, Ilyas, Antonicek, Schachner, and Quarles, 1986) and that in itself mediates adhesion (Künemund et al., 1988). In addition the filopodia as well as the entire growth cone of the fish retinal axons are $\mathrm{L} 2 / \mathrm{HNK}-1$ positive. The $\mathrm{L} 2 / \mathrm{HNK}-1$ positivity of the filopodia and membrane particles to which the filopodia adhered also suggests that filopodial-membrane contacts may be mediated, at least in part by this carbohydrate moiety and / or by cell adhesion molecules carrying this epitope. Cell adhesion molecules associated with fish retinal axons are N-CAM (Bastmeyer, Schlosshauer, and Stuermer, 1990) and two candidate cell adhesion molecules, the E 587 antigen (Vielmetter et al., 1991b) and Neurolin (Paschke, Lottspeich, and Stuermer, 1992), and probably more as suggested by ongoing studies. The involvement of strong adhesive interactions between filopodial tips and cell adhesion molecules on the membrane particles would explain why the filopodia remained attached to the membranes even when the growth cones had moved away from these sites or even when the growth cone had collapsed and retracted.

Filopodia in the current study, however, probably receive conflicting information on the presence of adhesive surfaces and strong repellent mole- 
cules. Unexplained remains the phenomenon that these filopodia persisted longer after contacts with membranes carrying the repellent guiding component in a high concentration than did those after contacts with other membranes or laminin. Such long-persisting filopodia were in the present study not seen with any other but the chick caudal membranes, although the membranes from rostral tectum and from caudal tectum of fish are likely to be of similar adhesivity as the chick caudal membranes. Consequently, it must be the combination of adhesion and strong repulsion mediated by the chick caudal membranes that evokes a longer than normal persistance of filopodia. It is conceivable that these filopodia adhering to the membranes containing the repellent component loose their force-generating ability. The loss of generating force could occur via different mechanisms as suggested by Mitchison and Kirschner (1988): by a loss of the linkage of surface receptors to the actin filaments; by an inhibition of the motor for retrograde movement of actin filaments; or by depolymerization of actin filaments.

The persistence of one filopodial strand on a retracting growth cone has previously been described by Kap fhammer and Raper (1987), when CNS growth cones met neurites of peripheral nervous system (PNS) origin. This adhering strand of a retracting growth cone even generated force on the PNS neurite so that the latter was observed to bend (Kap fhammer and Raper, 1987). Another difference between the retracting growth cones in Kap fhammer and Raper's study and the fish growth cones in the present study was that the fish growth cones upon retraction left behind not one but many filopodia and a substantial amount of material of the growth cone proper.

Walter et al. (1990) proposed that growth cones become paralyzed from interacting with the repellent component offered to the growth cone in a high concentration, however, whereas upon contact with the repellent component the forward growth is cancelled, other parts of the growth cone's machinery remain active. This is inferred from observing backward movement of particles along the persisting filopodia and all along the axons during retraction of the entire growth cone. Backward movement of particles was also observed in advancing growth cones and their filopodia. This supports the view that this kind of retrograde movement/transport is a normal event during growth cone progression. As demonstrated here, some kind of motor for backward particle movement appears to continue to work in persist- ing filopodia when the growth cone is repelled by the caudal membranes and during its collapse and retraction. Whether this is the same motor that is responsible for the retrograde movement of actin filaments (Mitchison and Kirschner, 1988) remains unsolved.

These findings may underscore the hypothesis (Cox et al., 1990; Walter et al., 1990) that the repellent component negatively affects specific parts of the growth cone's program required for forward growth, however, the normal growth program not only involves the production but also the retraction of lamellipodia and filopodia from sites from which the growth cone will turn away. The persistence of filopodia in the present experiments can be interpreted to mean that the repellent component also interferes with a mechanism that would normally result in filopodial retraction. The same may hold true for those parts of the growth cone that remain after entire growth cone retraction.

It would be necessary to know how the information is transmitted from the filopodium and lamellipodium and processed within the growth cone to fully account for the current growth cone behavior. The finding that contacts with a few filopodia and a narrow lamellipodial extension with the repellent component is enough to induce a turning or branching response suggests that each of these growth cone elements has a sufficient number of receptors on their surfaces to transmit relevant information on environmental cues into the growth cone proper. As long as most filo- and lamellipodia are on laminin the growth cone turns away from the direction from where the repellent stimulus was received and progresses. Growth cone progression is cancelled, however, if the majority of the filopodia and lamellipodia have encountered the repellent component with their tips, although the growth cone core remains on the laminin substrate.

At this point in our investigations it is impossible to define the membraneous or cytoskeletal elements of the filopodia that are transported in a backward fashion upon encounter with the repellent component and the ones that are remaining as strands after growth cone retraction. The L2/ HNK-1 and E 587 positivity of persisting filopodial strands of retracted and collapsed growth cones suggests that some membrane-associated cell adhesion molecules and, therefore, surface membranes may remain after growth cone collapse and withdrawal.

The behavior that fish retinal growth cones exhibit in response to the repellent guidance com- 
ponent of the tectal membranes differs from their reaction upon encountering mammalian CNS myelin and oligodendrocytes. Another earlier cross-species in vitro experiment has demonstrated that the mammalian CNS oligodendrocytes and myelin inhibitors (Schwab and Caroni, 1988) impair fish growth cone elongation (Bastmeyer, Beckmann, Schwab, and Stuermer, 1991). When meeting rat oligodendrocytes the fish growth cones remained on the cell surface for up to $2 \mathrm{~h}$ and exhibited extensive ruffling of lamellipodia during that time. The retraction and collapse that followed these interactions was rapid and adherent strands of filopodia or the growth cone core were never observed. In a similar dead end situation of the modified stripe assay as described here, fish growth cones grew onto rat CNS myelin fragments at the ends of the lanes and rarely collapsed on the myelin. Here again, ruffling of veils continued for hours, however, the growth cones hardly progressed, and instead moved in place like car wheels on ice. Together with the fact that all retinal axons whether from nasal or temporal retina reacted similarly to the myelin inhibitors (Bastmeyer et al., 1991), this difference in growth cone behavior revealed by time-lapse videomicroscopy may suggest that the repellent tectal component and the myelin inhibitors act through different receptors or intracellular signalling pathways.

The authors thank Anette Habring-Müller and Marianne Wiechers for excellent technical assistance.

\section{REFERENCES}

BAIER, H. and BoNHOEFFER, F. (1992). Axon guidance by gradients of a target-derived component. Science 255:472-475.

Bastmeyer, M., BeckManN, M., Schwab, M. E., and STUERMER, C. A. O. (1991). Growth of regenerating goldfish axons is inhibited by rat oligodendrocytes and CNS myelin but not by goldfish optic nerve tract oligodendrocytelike cells and fish CNS myelin. $J$. Neurosci. 11:626-640.

Bastmeyer, M., Schlosshauer, B., and Stuermer, C. A. O. (1990). The spatiotemporal distribution of N-CAM in the retinotectal pathway of adult goldfish detected by the monoclonal antibody D3. Development 108:299-311.

CoX, E. C., Müller, B., and BonhoefFer, F. (1990). Axonal guidance in the chick visual system: posterior tectal membranes induce collapse of growth cones from the temporal retina. Neuron 4:31-37.

DAvies, J. A. and Cook, G. M. W. (1991). Growth cone inhibition-an important mechanism in neural development. BioEssays 13:11-15.

GIERER, A. (1987). Directional cues for growing axons forming the retinotectal projection. Development 101:479-489.

Godement, P. and BonhoefFer, F. ( 1989). Cross-species recognition of tectal cues by retinal fibers in vitro. Development 106:313-320.

Jessell, T. M. ( 1988). Adhesion molecules and the hierarchy of neural development. Neuron 1:3-13.

Kaethner, R. J. and Stuermer, C. A. O. (1992). Dynamics of terminal arbor formation and target approach of retinotectal axons in living zebrafish embryos. A time lapse study of single axons. $J$. Neurosci. 12:3257-3277.

KAP FHAMMER, J. P. and RAPER, J. A. (1987). Collapse of growth cone structure on contact with specific neurites in culture. J. Neurosci. 7:201-212.

Künemund, V., Jungalwala, F. B., Fischer, G., Chou, D. K. H., Keilhauer, G., and Schachner, M. (1988). The L2/HNK-1 carbohydrate of neural cell adhesion molecules is involved in cell interactions. J. Cell Biol. 106:213-223.

Mitchison, T. and KirSChNer, M. (1988). Cytoskeletal dynamics and nerve growth. Neuron 1:761-772.

Müller, B., STAHL, B., and BONHOEFFER, F. (1990). In vitro experiments on axonal guidance and growthcone collapse. J. Exp. Biol. 153:29-46.

NorhoNa, A. B., Ilyas, A., ANTONICEK, H., Schachner, M., and Quarles, R. H. (1986). Molecular specificity of $\mathrm{L} 2$ monoclonal antibodies that bind to carbohydrate determinants of neural cell adhesion molecules and their resemblance to other monoclonal antibodies recognizing the myelin-associated glycoprotein. Brain Res. 385:237-244.

PASCHKe, K. A., LotTSPeich, F., and STUERmer, C. A. O. (1992). Neurolin, a cell surface glycoprotein on growing retinal axons in the goldfish visual system is re-expressed during retinal axonal regeneration. $J$. Cell Biol. 117:863-875.

Patterson, P. H. (1988). On the importance of being inhibited, or saying no to growth cones. Neuron 1:263-267.

Schlosshauer, B., Schwarz, U., and Rutishauser, U. (1984). Topological distribution of different forms of neural cell adhesion molecule in the developing chick visual system. Nature 310:141-143.

SCHWAB, M. E. and CARONI, P. (1988). Oligodendrocytes and CNS myelin are nonpermissive substrates for neurite growth and fibroblast spreading in vitro. $J$. Neurosci. 8:2381-2393.

Spectrum Videothek (1990). Gehirn und Nervensystem. ISBN 3-89330-966-7.

STUERMER, C. A. O. (1989). Dynamics of axonal growth and target recognition in the retinotectal system of fish. In: Dynamics and Plasticity in Neuronal Systems. N. Elsner and W. Singer, Eds. Thieme Verlag, Stuttgart/New York, pp. 87-92.

Stuermer, C. A. O., Bastmeyer, M., and Vielmet- 
TER, J. (1990). Avoidance response and collapse of fish temporal growth cones upon contact with caudal tectal membranes of embryonic chick. Soc. Neurosci. Abstr. 16:1126.

Vielmetter, J., WAlter, J., and Stuermer, C. A. O. (1991a). Regenerating retinal axons of goldfish respond to a repellent guiding component on caudal tectal membranes of adult fish and embryonic chick. $J$. Comp. Neurol. 311:321-329.

Vielmetter, J., LotTSPEICH, F., and Stuermer, C. A. O. (1991b). The monoclonal antibody E587 recognizes growing (new and regenerating) retinal axons in the goldfish retinotectal pathway. $J$. Neurosci. 11:3581-3593.

Vielmetter, J., Stolze, B., Bonhoeffer, F., and STUERMer, C. A. O. (1990). In vitro assay to test dif- ferential substrate affinities of growing axons and migratory cells. Exp. Brain Res. 81:283-287.

Vielmetter, J. and Stuermer, C. A. O. (1989). Goldfish retinal axons respond to position-specific properties of tectal cell membranes in vitro. Neuron 2:13311339.

WALTER, J., ALLSOPP, T. E., and BONHOEFFER, F. (1990). A common denominator of growth cone guidance and collapse. TINS 13:447-452.

Walter, J., Kern-Veits, B., Huf, J., Stolze, B., and BONHOEFFER, F. (1987a). Recognition of positionspecific properties of tectal cell membranes by retinal axons in vitro. Development 101:685-696.

WALTER, J., Henke-Fahle, S., and BonhoefFER, F. (1987b). Avoidance of posterior tectal membranes by temporal retinal axons. Development 101:909-913. 